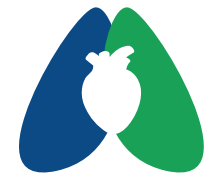

ASSOBRAFIR C I Ê N C I A

\section{Equações de referência disponíveis mundialmente para o pico de fluxo expiratório em crianças e adolescentes: uma revisão sistemática}

\author{
Reference equations available worldwide for the peak of expiratory \\ flow in children and adolescents: a systematic review
}

Maria Amélia Bagatini (1), Daniele Schiwe ${ }^{1}$ (), João Paulo Heinzmann-Filho²* (1)
'Pontifícia Universidade Católica do Rio Grande do Sul (PUCRS), Porto Alegre, RS, Brasil

${ }^{2}$ Centro Universitário Cenecista de Osório (UNICNEC), Osório, RS, Brasil

Como citar: Bagatini MA, Schiwe D, Heinzmann-Filho JP. Equações de referência disponíveis mundialmente para o pico de fluxo expiratório em crianças e adolescentes: uma revisão sistemática. ASSOBRAFIR Ciênc. 2022;13:e424897. https://doi.org/10.47066/2177-9333. AC. 2020.0045

Submissão em: Janeiro 21, 2021 Aceito em: Dezembro 24, 2021

Estudo realizado em: UNICNEC, Osório, RS, Brasil.

Aprovação ética: Não se aplica.

*Autor correspondente: João Paulo Heinzmann-Filho. E-mail: joaopauloheinzmann@hotmail.com

\begin{abstract}
Resumo
Introdução: Existem diversas equações de referência para o pico de fluxo expiratório (PFE) na população pediátrica. Objetivo: Revisar equações de referência disponíveis na literatura científica para o PFE em crianças e adolescentes. Métodos: Trata-se de uma revisão sistemática, conduzida nas bases de dados PubMed, Scielo, Lilacs e PEDro. Foram incluídos estudos que geraram equações de referência ao redor do mundo para o PFE em crianças e adolescentes saudáveis. A estratégia de busca foi baseada na combinação dos descritores: Reference Values; Normal Value; Peak Expiratory Flow; Maximum expiratory flow; Pediatrics. A qualidade metodológica foi avaliada pela escala Quality Assessment of Diagnostic Accuracy Studies. Resultados: De um total de 487 artigos, apenas 13 foram incluídos. Grande parte deles foi conduzida (7/13) no continente Asiático. As amostras totalizaram 14.969 participantes (idade: 4-18 anos). O exame clínico (7/13) foi o método mais frequente para a definição do status de saudável. Quase todos eles (9/13) utilizaram o equipamento MiniWright ${ }^{\circledR}$, realizando até 03 mensurações (9/13) e utilizando o maior valor obtido de PFE (10/13). Embora exista oscilação nas fórmulas preditivas, 13/13 estudos apresentaram as equações baseadas na altura e 11/13 separadas pelo sexo. As fórmulas de referência explicaram entre $18 \%$ e $76 \%$ os valores do PFE, obtendo as melhores predições nos trabalhos do Continente Asiático. A qualidade metodológica foi considerada adequada (12/13). Conclusão: Apesar da variabilidade observada nas equações de referência, a altura e sexo apresentaram maior influência na predição dos valores do PFE. Ainda se fazem necessários valores de referência em diversas localidades do mundo.
\end{abstract}

Palavras-chave: Testes de Função Respiratória; Pico de Fluxo Expiratório; Valores de Referência; Pediatria.

\begin{abstract}
Backgound: There are several reference equations for peak expiratory flow (PEF) in the pediatric population. Aim: To review reference equations available in the scientific literature for PEF in children and adolescents. Methods: This is a systematic review, conducted in PubMed, Scielo, Lilacs and PEDro databases. Studies that generated reference equations around the world for PEF in healthy children and adolescents were included. Search strategy was based on the combination of descriptors: Reference Values; Normal Value; Peak Expiratory Flow; Maximum expiratory flow; Pediatrics. Methodological quality was assessed using the Quality Assessment of Diagnostic Accuracy Studies scale. Results: From a total of 487 articles, only 13 were included. Most of them were conducted (7/13) on the Asian continent. The samples totaled 14,969 participants (age: 4-18 years). Clinical examination (7/13) was the most frequent method for defining healthy status. Almost all of them (9/13) used the Mini-Wright ${ }^{\circledR}$ equipment, performing up to 03 measurements $(9 / 13)$ and using the highest PEF value obtained (10/13). Although there is oscillation in the predictive formulas, $13 / 13$ studies presented the equations based on height and 11/13 separated by sex. The reference formulas explained between $18 \%$ and $76 \%$ of the PEF values, obtaining the best predictions in the works from the Asian continent. The methodological quality was considered adequate (12/13). Conclusion: Despite the variability observed in the reference equations, height and sex had a greater influence on the prediction of PEF values. Reference values are still needed in different parts of the world.
\end{abstract}

Keywords: Respiratory Function Tests; Peak Expiratory Flow; Reference Values; Pediatrics. 


\section{INTRODUÇÃO}

O pico de fluxo expiratório (PFE) é um dos testes de função pulmonar que avalia o maior fluxo de ar registrado em uma expiração forçada a partir da capacidade pulmonar total ${ }^{1}$. As medidas deste método podem ser realizadas através de espirômetro ou baseando-se em dispositivo manual portátil ${ }^{1,2}$.

O PFE vem ganhando cada vez mais espaço no ambiente hospitalar e ambulatorial, visto que apresenta papel essencial na avaliação da efetividade da tosse $e^{2,3}$. A magnitude do fluxo expiratório, gerado no momento da tosse ou em uma expiração forçada, é o principal fator responsável pela clearance mucociliar. Fluxos elevados podem ser obtidos com labilidade brônquica adequada, força muscular e volume pulmonar preservado e/ou ausência de secreção nas vias aéreas ${ }^{3,4}$.

Estudos prévios vêm demonstrando o papel do PFE no diagnóstico e na identificação de exacerbação da asma na população pediátrica ${ }^{5-8}$, além de sua aplicabilidade no processo de desmame nas doenças neuromusculares ${ }^{9,10}$. Aumentos de 15\% no fluxo expiratório máximo após o uso de broncodilatador, administração de corticosteróide ou variação diurna (>20\%) ao longo de três semanas, podem ser sinais indicativos de asma ${ }^{7}$. Também, quedas maiores de $50 \%$ do fluxo predito ou do melhor resultado conhecido, constitui-se um sinal de maior gravidade da exacerbação da doença ${ }^{8}$. Além disso, resultados abaixo de 160 litros/ minuto estão relacionados com insucesso na extubação ou decanulação ${ }^{9}$ e valores menores que 60 litros/minuto apresentam maiores riscos de falha na extubação em doenças neuromusculares ${ }^{10}$.

Uma das formas de interpretação do resultado obtido no PFE ocorre a partir da análise do percentual do previsto. Este processo é realizado através de equações de referência, comumente baseadas em amostras saudáveis ${ }^{2,11}$. Atualmente, existem diversas fórmulas preditivas em crianças e adolescentes ao redor do mundo ${ }^{12-24}$, nestas, a idade, altura e o sexo parecem estimar adequadamente os fluxos expiratórios máximos. No entanto, há divergências quanto à metodologia utilizada, equações geradas e oscilação no poder de predição de cada estudo ${ }^{12-24}$. Além disso, existem países que não apresentam equações de referência para o PFE.

Portanto, é necessário realizar um mapeamento das equações existentes para o PFE na população pediátrica, buscando direcionar o uso da fórmula mais adequada em cada localidade e também observar possíveis lacunas sobre o tema. Além do mais, não foi localizada nenhuma revisão sistemática sobre o assunto, justificando o desenvolvimento deste estudo. Assim, o objetivo deste trabalho foi revisar as equações de referência disponíveis na literatura científica para o PFE em crianças e adolescentes.

\section{MÉTODOS}

A revisão sistemática foi realizada de acordo com as recomendações da Preferred Reporting Items for Systematic Reviews e Meta-Analyzes ${ }^{25}$ e registrada no PROSPERO sob protocolo CRD42020184658.

\section{Fonte de dados}

Utilizaram-se as bases de dados PubMed via Medline, Literatura Latino-Americana e do Caribe em Ciências da Saúde (Lilacs), Scientific Electronic Library Online (SCIELO) e Physiotherapy Evidence Database (PEDro).

\section{Estratégia de busca}

A busca utilizada para a seleção dos artigos foi baseada em cinco palavras-chave, associadas com dois diferentes operadores booleanos. Utilizou-se a seguinte estratégia de busca nas plataformas PubMed e SciELO: Reference Values OR Normal Values AND Peak Expiratory Flow OR Maximum expiratory flow AND Pediatrics. Já nas plataformas Lilacs e PEDro não foi utilizado o descritor "Pediatrics" devido à ausência de pesquisas localizadas sobre o tema. Nestas bases de dados, a estratégia de busca foi a seguinte: Reference Values OR Normal Values AND Peak Expiratory Flow OR Maximum expiratory flow.

Todos esses termos são descritores controlados, cadastrados nos Descritores em Ciências da Saúde (DECS) e também registrados no Entry Terms, com exceção das palavras "Peak Expiratory Flow" " "Maximum expiratory flow". Optou-se por deixá-las pelo fato de terem relação direta com o tema e também pelos estudos utilizarem esses termos no resumo (abstract).

Estes descritores deveriam constar, pelo menos, no título, no resumo ou nas palavras-chave. Além disso, foram revisadas as referências dos estudos incluídos para verificar possíveis artigos que pudessem compor o presente trabalho.

\section{Seleção dos estudos e elegibilidade}

Foram incluídos estudos observacionais que geraram equações de referência ao redor do mundo para o PFE em crianças e adolescentes (até 18 anos) saudáveis, de acordo com critérios estabelecidos por cada autor. Em contrapartida, excluíram-se estudos de revisão, pesquisas que geraram valores de referência para o PFE utilizando a espirometria, artigos sem relação com o tema, amostras adultas (acima de 18 anos de idade), estudos repetidos, pesquisas com doenças associadas e documentos indisponíveis.

Não foram utilizados filtros em relação ao idioma e ao ano de publicação das pesquisas. A seleção dos artigos ocorreu em abril e maio de 2020. 


\section{Extração de dados}

Todos os artigos encontrados passaram pela leitura dos títulos e/ou leitura dos resumos para avaliar a adequação quanto aos critérios de elegibilidade. Os estudos que apresentaram os critérios predeterminados tiveram o texto completo adquirido para análise e extração dos dados. A busca e a análise dos artigos foram conduzidas de forma independente, por dois avaliadores (MAB e JPHF), sendo qualquer divergência resolvida por consenso.

Foram coletadas as seguintes características dos estudos: nome do primeiro autor, ano de publicação, país da coleta de dados, número de participantes, tipo de seleção da amostra, cálculo amostral, faixa etária, condição socioeconômica, localização, método de avaliação para o status de saudável, modelo do equipamento utilizado, quantidade de medidas e valor registrado do $\mathrm{PFE}$, além das equações de referência e do coeficiente de determinação $\left(R^{2}\right)$.

\section{Avaliação da qualidade metodológica}

A avaliação da qualidade metodológica foi realizada por dois pesquisadores, de forma independente, sendo qualquer divergência resolvida por consenso. A análise foi conduzida por meio da escala Quality Assessment of Diagnostic Accuracy Studies (QUADAS-2) ${ }^{26}$. Esta ferramenta permite avaliação do risco de viés (baixo, alto ou incerto) na seleção de paciente, teste avaliado, padrão de referência, fluxo/tempo, além da preocupação quanto a aplicabilidade (baixa, alta ou incerta) nos dois primeiros domínios. O item padrão de referência não foi analisado nesta revisão, visto que não se aplica ao objetivo do estudo, corroborando com outros trabalhos ${ }^{27-29}$. O sistema de análise foi similar ao divulgado por uma revisão sistemática ${ }^{29}$, com diferença apenas no teste avaliado, ou seja, no direcionamento para o $\mathrm{PFE}^{30}$. A qualidade metodológica foi considerada adequada quando $\geq 3$ (total de cinco pontos). Avaliaram-se outros aspectos adicionais, incluindo a representatividade (>10 participantes por faixa etária) ${ }^{30}$, validação cruzada ${ }^{31}$, poder de predição e erro conhecido nas fórmulas previstas.

\section{RESULTADOS}

De um total de 487 artigos, 414 foram encontrados no Pubmed, 42 na SciELO, 27 no Lilacs e 04 no PEDro. Destes, 42 foram excluídos por estarem repetidos nas bases de dados, restando 445 documentos. Após, 434 foram descartados pelo fato de não preencherem os critérios de elegibilidade, permanecendo 11 pesquisas. Em adição, mais dois artigos foram selecionados através da busca de pesquisas nas referências dos estudos incluídos. A presente revisão foi composta de 13 artigos (Figura 1).

Dos 13 estudos incluídos, oito (61,5\%) foram conduzidos no continente Asiático, três (23,0\%) na Europa, um na América do Sul (7,7\%) e um na África (7,7\%). No total, foram selecionados 14.969 participantes, com tamanho

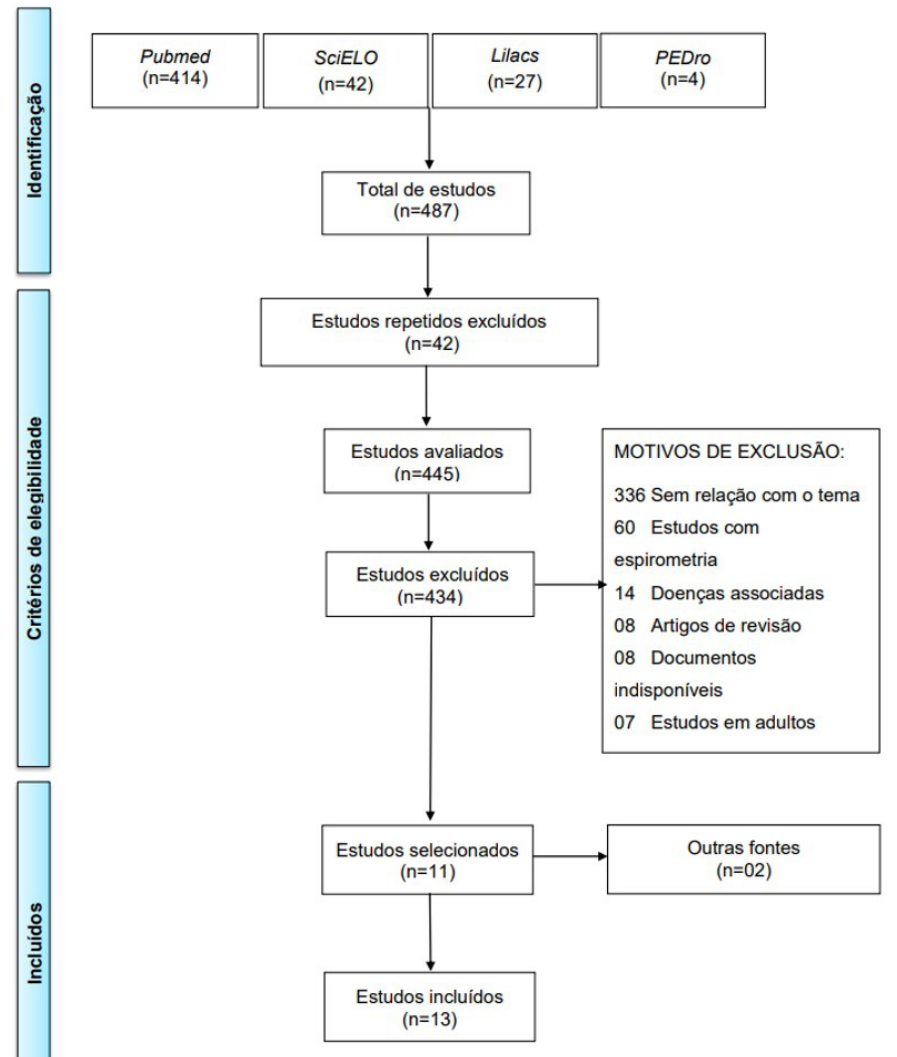

Figura 1. Sistematização da seleção dos estudos nessa revisão.

amostral oscilando de 340 a 3.169 sujeitos. Mais da metade dos trabalhos $(53, \%)$ selecionaram seus participantes randomicamente e apenas um $(7,7 \%)$ reportou o cálculo de tamanho amostral (Tabela 1).

A idade dos indivíduos variou de quatro a 18 anos, com predomínio do sexo feminino $(\mathrm{N}=7.613)$. Apenas cinco pesquisas $(38,4 \%)$ reportaram a condição socioeconômica, predominando a baixa renda $(60,0 \%)$. Nove documentos $(69,2 \%)$ descreveram a localização dos participantes, sendo a maior parte $(55,5 \%)$ deles oriundos da zona urbana. Além disso, a definição do status de saudável foi baseada no exame clínico $(53,8 \%)$ e no histórico de sintomas respiratórios (23,0\%). Nenhum estudo incluiu participantes com doenças pré-existentes (Tabela 2).

Quase todos os estudos selecionados (69,2\%) utilizaram o equipamento Mini-Wright ${ }^{\circledR}$, com a grande maioria $(69,2 \%)$ realizando até 03 mensurações e utilizando o maior valor (76,9\%) nestes registros (Tabela 3). Apesar de existir variabilidade nas fórmulas preditivas, a altura foi a variável preditora mais frequente (100\%), seguida do peso $(38,5 \%)$ e da idade (30,8\%). Ainda, $84,6 \%$ das pesquisas apresentaram as equações de referência separadas pelo sexo. Apenas seis estudos (46,1\%) reportaram o coeficiente de determinação de suas equações, explicando entre $18 \%$ e $76 \%$ os resultados do PFE. Os trabalhos do continente Asiático se destacaram pelo maior poder de predição (Tabela 4). 
Tabela 1. Identificação dos estudos e descrição da amostragem realizada nos trabalhos incluídos nesta revisão.

\begin{tabular}{|c|c|c|c|c|}
\hline Primeiro autor \& ano & País & $\mathbf{N}$ de participantes & $\begin{array}{c}\text { Tipo de seleção da } \\
\text { amostra }\end{array}$ & Cálculo amostral \\
\hline Lu et al. (2018) & China & 3169 & Randomizada & Não \\
\hline Kumaran et al. (2017) ${ }^{13}$ & Índia & 2156 & Conveniência & Sim \\
\hline Pramanik et al. (2014) ${ }^{14}$ & Índia & 1288 & Randomizada & Não \\
\hline Manjunath et al. (2013) & Índia & 1008 & Conveniência & Não \\
\hline Taksande et al. (2008) $)^{16}$ & Índia & 1078 & Randomizada & Não \\
\hline Boaventura et al. (2007) ${ }^{17}$ & Brasil & 1024 & Randomizada & Não \\
\hline Agaba et al. (2003) $)^{18}$ & Nigéria & 1025 & Randomizada & Não \\
\hline Høst et al. (1994) $)^{19}$ & Dinamarca & 861 & Conveniência & Não \\
\hline Swaminathan et al. (1993) ${ }^{20}$ & Índia & 345 & Randomizada & Não \\
\hline Kashyap et al. (1992) ${ }^{21}$ & Índia & 237 & Conveniência & Não \\
\hline Sanz et al. $(1990)^{22}$ & Espanha & 1566 & Randomizada & Não \\
\hline Pande $(1986)^{23}$ & Índia & 350 & Conveniência & Não \\
\hline \multirow[t]{2}{*}{ Tsanakas et al. $(1983)^{24}$} & Grécia & 522 & \multirow{2}{*}{ Conveniência } & \multirow[t]{2}{*}{ Não } \\
\hline & Inglaterra & 340 & & \\
\hline
\end{tabular}

N: Número.

Por fim, 12/13 (92,3\%) estudos foram classificados com qualidade metodológica adequada, obtendo uma média de 3,5 pontos (variação: 1-5). Os fatores que baixaram predominantemente esta análise foram relativos ao risco de viés e a preocupação quanto à aplicabilidade do teste avaliado, além do risco de viés no aspecto fluxo/ tempo (Tabela 5). Embora, todas as pesquisas obtiveram amostras representativas, nenhuma testou a validação cruzada, somente cinco relataram o poder de predição e duas delas divulgaram os erros conhecidos nas equações de referência.

\section{DISCUSSÃO}

A presente revisão realizou um mapeamento das equações de referência disponíveis para a normalização e interpretação dos resultados no PFE em amostras pediátricas (4-18 anos), baseando-se em 13 pesquisas ${ }^{12-24}$ ao redor do mundo. Este trabalho pode servir como um guia terapêutico para a tomada de decisão, considerando a análise das fórmulas preditivas disponíveis na literatura científica para diversas localidades. Nestas pesquisas, embora, se observou variabilidade nas equações preditivas, percebeu-se que a altura e o sexo foram as variáveis que mais contribuíram para a predição dos resultados do PFE. Além disso, as fórmulas existentes explicaram entre $18 \%$ e $76 \%$ os valores deste desfecho, destacando-se o maior poder predição dos estudos publicados no Continente Asiático ${ }^{12-14,20}$.

A maioria dos estudos encontrados nesta revisão é de origem Asiática ${ }^{12-16,20,21,23}$, sendo conduzidos predominantemente na Índia $13-16,20,21,23$. Houve também investigações em outras localidades ${ }^{17-19,22,24}$, exceto nos continentes Oceânicos e nas Américas do Norte e Central. Esses resultados demonstram a necessidade de valores de normalidade para o PFE nestas últimas, já que a American Thoracic Society (ATS) recomenda a escolha de equações de referência para cada região ${ }^{32}$. O Brasil, por exemplo, é um país da América Latina de dimensão continental, de grande diversidade étnica e climática, portanto, cada localidade do mesmo pode ter diferentes estimativas de PFE e assim ocasionar sub/superestimação dos resultados ${ }^{17,33}$. Segundo os achados da nossa pesquisa, apenas um trabalho foi realizado no Brasil, sendo este conduzido em Minas Gerais, nas cidades de Uberlândia e Araguari ${ }^{17}$. Com isso, recomenda-se a geração de valores de referência a partir de pesquisa multicêntrica para a maior representatividade do país. Além do mais, este trabalho nacional ${ }^{17}$ não reportou o coeficiente de determinação $\left(R^{2}\right)$ de suas equações, inviabilizando assim, a reflexão sobre a real capacidade de predição desta fórmula.

Embora grande parte das pesquisas selecionadas utilizaram as variáveis altura ${ }^{12-24}$ e sexo $0^{12-17,19-23}$ para a predição do PFE em crianças e adolescentes, as fórmulas preditivas apresentaram variabilidade. A altura pode interferir nos resultados do PFE, já que a complacência torácica e pulmonar sofre alterações com o avançar da idade. Relatos apontam que o pico de transformação anatômica ocorre na fase de estirão da criança (8 aos 14 anos), gerando ganhos nos volumes pulmonares ${ }^{34-37}$. Além disso, sabe-se que os valores de PFE dos meninos são mais elevados em relação ao sexo feminino. Isso 
Tabela 2. Características sociodemográficas dos indivíduos recrutados em cada estudo.

\begin{tabular}{|c|c|c|c|c|c|}
\hline Primeiro autor $\&$ ano & Sexo & Faixa etária (anos) & $\begin{array}{c}\text { Condição } \\
\text { socioeconômica }\end{array}$ & Localização & $\begin{array}{l}\text { Definição do } \\
\text { status de saudável }\end{array}$ \\
\hline Lu et al. (2018) $)^{12}$ & $\begin{array}{l}1.573 \mathrm{~F} \\
1.596 \mathrm{M}\end{array}$ & $5-14$ & - & Zona urbana & $\begin{array}{l}\text { Exame clínico } \\
\text { e questionário } \\
\text { inespecífico }\end{array}$ \\
\hline Kumaran et al. (2017) $)^{13}$ & $\begin{array}{l}1.399 \mathrm{~F} \\
757 \mathrm{M}\end{array}$ & $10-16$ & - & Zona urbana & $\begin{array}{l}\text { Exame clínico e } \\
\text { questionário criado } \\
\text { pelos autores }\end{array}$ \\
\hline Pramanik et al. (2014) $)^{14}$ & $\begin{array}{l}521 \mathrm{~F} \\
767 \mathrm{M}\end{array}$ & $13-18$ & - & - & Exame clínico \\
\hline $\begin{array}{l}\text { Manjunath et al. } \\
\qquad(\mathbf{2 0 1 3})^{15}\end{array}$ & $\begin{array}{l}437 \mathrm{~F} \\
571 \mathrm{M}\end{array}$ & $5-16$ & Baixa renda* & Zona rural & $\begin{array}{l}\text { Exame clínico } \\
\text { baseado na ATS }\end{array}$ \\
\hline Taksande et al. (2008) & $\begin{array}{l}589 \mathrm{~F} \\
489 \mathrm{M}\end{array}$ & $8-15$ & Baixa renda* & Zona rural & $\begin{array}{l}\text { Histórico baseado } \\
\text { em sintomas } \\
\text { respiratórios }\end{array}$ \\
\hline $\begin{array}{l}\text { Boaventura et al. } \\
\qquad(2007)^{17}\end{array}$ & $\begin{array}{l}501 \mathrm{~F} \\
523 \mathrm{M}\end{array}$ & $12-18$ & $\begin{array}{c}\text { Alta, média e baixa } \\
\text { renda }\end{array}$ & Zona urbana & $\begin{array}{l}\text { Histórico baseado } \\
\text { em sintomas } \\
\text { respiratórios }\end{array}$ \\
\hline Agaba et al. (2003) & $\begin{array}{l}495 \mathrm{~F} \\
530 \mathrm{M}\end{array}$ & $6-12$ & - & Zona urbana & $\begin{array}{l}\text { Histórico baseado } \\
\text { em sintomas } \\
\text { respiratórios }\end{array}$ \\
\hline Høst et al. (1994) ${ }^{19}$ & $\begin{array}{l}439 \mathrm{~F} \\
422 \mathrm{M}\end{array}$ & $6-17$ & - & Zona rural & $\begin{array}{l}\text { Questionário criado } \\
\text { pelos autores }\end{array}$ \\
\hline $\begin{array}{l}\text { Swaminathan et al. } \\
(1993)^{20}\end{array}$ & $\begin{array}{l}191 \mathrm{~F} \\
154 \mathrm{M}\end{array}$ & $4-15$ & Baixa renda* & - & Exame clínico \\
\hline Kashyap et al. (1992) & $\begin{array}{c}98 \mathrm{~F} \\
139 \mathrm{M}\end{array}$ & $7-14$ & - & Zona urbana & Exame clínico \\
\hline Sanz et al. $(1990)^{22}$ & $\begin{array}{l}774 \mathrm{~F} \\
792 \mathrm{M}\end{array}$ & $7-14$ & - & - & $\begin{array}{l}\text { Questionário } \\
\text { sobre sintomas } \\
\text { respiratórios da } \\
\text { ATS }\end{array}$ \\
\hline Pande $(1986)^{23}$ & $\begin{array}{l}175 \mathrm{~F} \\
175 \mathrm{M}\end{array}$ & $5-12$ & - & Zona rural & - \\
\hline Tsanakas et al. (1983) & $\begin{array}{c}266 \mathrm{~F} \\
256 \mathrm{M} \\
155 \mathrm{~F} \\
185 \mathrm{M}\end{array}$ & $7-16$ & $\begin{array}{l}\text { Intermediária } \\
\text { renda* }\end{array}$ & - & Exame clínico \\
\hline
\end{tabular}

F: Feminino. M: Masculino. ATS: American Toracic Society. -: Não informado. *Maior parte da amostra reportada.

parece ser atribuído pelo fato de que o sexo masculino apresenta melhor condição muscular torácica 17,18,33,34. Apesar de, fisiologicamente, a idade possuir relação com o desenvolvimento da árvore brônquica, além do peso ter associação com o tamanho e a forma da caixa torácica ${ }^{37}$, poucos estudos incluíram estes parâmetros nas equações preditivas ${ }^{13,14,16,17,19,20,23}$. Ainda que seis estudos $2-14,16,18,20$ reportaram o poder de predição ( $\left.R^{2}: 18-76 \%\right)$ de suas equações de referência, somente as pesquisas conduzidas na $\mathrm{China}^{12}$ e na
Índia ${ }^{13,14,20}$ conseguiram prever adequadamente $\left(R^{2}>0,70\right)$ os valores de PFE. Fatores como maturação sexual e nível de atividade física podem ter influenciado nestes achados, porém, não foram levados em consideração nos trabalhos.

Quase todos os trabalhos selecionados utilizaram o equipamento Mini-Wrigth ${ }^{13,15,16,19-24}$. Este dispositivo é considerado confiável e de fácil aplicabilidade, por isso vem ganhando espaço em laboratórios especializados, ambulatórios e hospitais ${ }^{3}$. No âmbito científico, tem sido utilizado em diversas condições clínicas 
Tabela 3. Características metodológicas e principais resultados do PFE.

\begin{tabular}{|c|c|c|c|}
\hline Primeiro autor \& ano & Modelo de PFE & $\mathrm{N}^{\circ}$ de registros & PFE utilizado \\
\hline Lu et al. $(2018)^{12}$ & Mini peak flow meter Airmed® & 06 & - \\
\hline Kumaran et al. (2017) & Mini-Wright ${ }^{\circledR}$ & llimitado\# & Maior valor alcançado \\
\hline Pramanik et al. (2014) & Mini peak flow meter Airmed® & 03 & Maior valor alcançado \\
\hline Manjunath et al. (2013) & Mini-Wright ${ }^{2}$ & 05 & Média dos registros* \\
\hline Taksande et al. (2008) ${ }^{16}$ & Mini-Wright ${ }^{2}$ & 03 & Maior valor alcançado \\
\hline Boaventura et al. (2007) ${ }^{17}$ & Healthscan Products Inc & 03 & Maior valor alcançado \\
\hline Agaba et al. $(2003)^{18}$ & Peak flow meter & 03 & Maior valor alcançado \\
\hline Høst et al. (1994) & Mini-Wright ${ }^{\circledR}$ & 03 & Maior valor alcançado \\
\hline Swaminathan et al. $(1993)^{20}$ & Mini-Wright $®$ & 03 & Maior valor alcançado \\
\hline Kashyap et al. (1992) & Mini-Wright ${ }^{\circledR}$ & 03 & Maior valor alcançado \\
\hline Sanz et al. $(1990)^{22}$ & Mini-Wright ${ }^{\circledR}$ & 03 & Maior valor alcançado \\
\hline Pande $(1986)^{23}$ & Mini-Wright ${ }^{\circledR}$ & 03 & Média dos registros \\
\hline Tsanakas et al. $(1983)^{24}$ & Mini-Wright ${ }^{\circledR}$ & 05 & Maior valor alcançado \\
\hline
\end{tabular}

-: Não informado. \#: Quantidade necessária até atingir 03 medidas reprodutíveis (20L/min). *: Média dos 03 maiores valores registrados.

Tabela 4. Descrição das fórmulas preditivas e do coeficiente de determinação $\left(R^{2}\right)$.

\begin{tabular}{|c|c|c|}
\hline Primeiro autor \& ano & Equações de referência & $\mathbf{R}^{2}$ \\
\hline \multirow{2}{*}{ Lu et al. (2018) ${ }^{12}$} & $F=4,13 \times$ altura $(\mathrm{cm})-278,04$ & \multirow{2}{*}{$0,72-0,76$} \\
\hline & $M=4,39 \times$ altura $(\mathrm{cm})-300,48$ & \\
\hline \multirow{2}{*}{ Kumaran et al. (2017) ${ }^{13}$} & $F=-486,544+1,064 \times$ peso $(\mathrm{kg})+462,282+$ altura $(\mathrm{m})$ & \multirow{2}{*}{$0,72-0,73$} \\
\hline & $M=-464+1,408 \times$ peso $(\mathrm{kg})+439,192+$ altura $(\mathrm{m})$ & \\
\hline \multirow{3}{*}{ Pramanik et al. (2014) $)^{14}$} & $F=(19,64 \times$ idade $[$ anos $])-(0,0988 \times$ altura $[\mathrm{cm}])+32,455 \ddagger$ & \multirow{3}{*}{$0,59-0,76$} \\
\hline & $\mathrm{F}=(20,580 \times$ idade $[$ anos] $)-(0,2486 \times$ peso $[\mathrm{kg}])+19,248-6.671 \ddagger$ & \\
\hline & $M=(18,584 \times$ idade $[$ anos $])+(3,364 \times$ altura $[\mathrm{cm}])-387,584 \ddagger$ & \\
\hline \multirow{3}{*}{ Manjunath et al. (2013) ${ }^{15}$} & $M=(21,812 \times$ idade $[$ anos $])+(2,169 \times$ peso $[\mathrm{kg}])-6,671 \ddagger$ & \\
\hline & $F=4,25 \times$ altura $(\mathrm{cm})-321,21$ & \\
\hline & $M=4,40 \times$ altura $(\mathrm{cm})-317,42$ & \\
\hline \multirow{3}{*}{ Taksande et al. (2008) ${ }^{16}$} & $F=3,64 x$ altura $(\mathrm{cm})-257,86$ & \multirow{3}{*}{$0,18-0,38$} \\
\hline & $F=4,27 \times$ peso $(\mathrm{kg})+124,42$ & \\
\hline & $M=4,7 \times$ altura $(\mathrm{cm})-346,51$ & \\
\hline \multirow{3}{*}{ Boaventura et al. (2007) $)^{17}$} & $M=5,6 \times$ peso $(\mathrm{kg})+159,37$ & \\
\hline & $F=212,82 \times$ altura $(m)+(5,93 \times$ idade $[a n o s])-131,81$ & \multirow{2}{*}{-} \\
\hline & $M=295,79 \times$ altura $(m)+(24,96 \times$ idade $[a n o s])-478,24$ & \\
\hline Agaba et al. (2003) $)^{18}$ & $3,45 \times$ altura $(\mathrm{cm})-222$ & 0,60 \\
\hline \multirow{2}{*}{ Høst et al. (1994) $)^{19}$} & $F=-(2,2 \times$ altura $[\mathrm{cm}])+(14,2 \times$ idade $[$ anos $])-143,9$ & \\
\hline & $M=-(3,8 \times$ altura $[\mathrm{cm}])+(10,6 \times$ idade $[$ anos $])-313,2$ & \\
\hline \multirow{2}{*}{ Swaminathan et al. $(1993)^{20}$} & $\mathrm{~F}=2,03 \times$ altura $(\mathrm{cm})+3,18$ idade $($ anos $)+2,71$ peso $(\mathrm{kg})-132,92 \ddagger$ & \multirow{2}{*}{0,75} \\
\hline & $\mathrm{M}=2,04 \times$ altura $(\mathrm{cm})+4,78$ idade $($ anos $)+2,73$ peso $(\mathrm{kg})-134,29 \ddagger$ & \\
\hline
\end{tabular}

-: Não informado. t: Regressão trivariada escolhida para a tabela. ł: Regressão multivariada escolhida para a tabela. \#: Regressão simples escolhida para a tabela. $\mathrm{R}^{2}$ : Coeficiente de determinação. 
Tabela 4. Continuação...

\begin{tabular}{cc}
\hline Primeiro autor \& ano & Equações de referência \\
\hline Kashyap et al. (1992) ${ }^{21}$ & $\mathrm{~F}=-354,1+4,41 \times$ altura $(\mathrm{cm})$ \\
Sanz et al. (1990) ${ }^{22}$ & $\mathrm{M}=-464,07+5,07 \times$ altura $(\mathrm{cm})$ \\
Pande $(\mathbf{1 9 8 6})^{23}$ & $\mathrm{~F}=5,20 \times$ altura $(\mathrm{cm})-386,58^{\#}$ \\
& $\mathrm{M}=4,99 \times$ altura $(\mathrm{cm})-346,59^{\#}$ \\
& $\mathrm{~F}=-181,9688+9,4645$ peso $(\mathrm{kg})+1,15544$ altura $(\mathrm{cm}) \dagger$ \\
Tsanakas et al. (1983) $)^{24}$ & Grécia: $5,34 \times$ altura $(\mathrm{cm})-380,8$ \\
& Inglaterra: $5,64 \times$ altura $(\mathrm{cm})-472,5$
\end{tabular}

-: Não informado. †: Regressão trivariada escolhida para a tabela. ł: Regressão multivariada escolhida para a tabela. \#: Regressão simples escolhida para a tabela. $\mathrm{R}^{2}$ : Coeficiente de determinação.

Tabela 5. Avaliação da qualidade metodológica dos artigos incluídos.

\begin{tabular}{|c|c|c|c|c|c|c|}
\hline \multicolumn{7}{|c|}{ Escala QUADAS-2 } \\
\hline \multirow[b]{2}{*}{ Primeiro autor \& ano } & \multicolumn{3}{|c|}{ Risco de viés } & \multicolumn{2}{|c|}{ Preocupação de aplicabilidade } & \multirow{2}{*}{$\begin{array}{c}\text { Total } \\
05 \text { pontos) }\end{array}$} \\
\hline & $\begin{array}{l}\text { Seleção de } \\
\text { paciente }\end{array}$ & Teste avaliado & Fluxo \& tempo & $\begin{array}{l}\text { Seleção de } \\
\text { paciente }\end{array}$ & Teste avaliado & \\
\hline Lu et al. $(2018)^{12}$ & Baixo & Incerto & Baixo & Baixa & Incerta & 03 \\
\hline Kumaran et al. $(2017)^{13}$ & Baixo & Incerto & Baixo & Baixa & Incerta & 03 \\
\hline $\begin{array}{l}\text { Pramanik et al. } \\
(2014)^{14}\end{array}$ & Baixo & Baixo & Alto & Baixa & Baixa & 04 \\
\hline $\begin{array}{l}\text { Manjunath et al. } \\
(2013)^{15}\end{array}$ & Baixo & Alto & Baixo & Baixa & Alta & 03 \\
\hline $\begin{array}{l}\text { Taksande et al. } \\
(2008)^{16}\end{array}$ & Baixo & Incerto & Baixo & Baixa & Incerta & 03 \\
\hline $\begin{array}{l}\text { Boaventura et al. } \\
\qquad(2007)^{17}\end{array}$ & Baixo & Baixo & Baixo & Baixa & Baixa & 05 \\
\hline Agaba et al. $(2003)^{18}$ & Baixo & Incerto & Baixo & Baixa & Incerta & 03 \\
\hline Høst et al. (1994) $)^{19}$ & Baixo & Baixo & Alto & Baixa & Baixa & 04 \\
\hline $\begin{array}{l}\text { Swaminathan et al. } \\
\qquad(1993)^{20}\end{array}$ & Baixo & Baixo & Baixo & Baixa & Baixa & 05 \\
\hline Kashyap et al. (1992)21 & Baixo & Incerto & Baixo & Baixa & Incerta & 03 \\
\hline Sanz et al. $(1990)^{22}$ & Baixo & Baixo & Baixo & Baixa & Baixa & 05 \\
\hline Pande $(1986)^{23}$ & Incerto & Alto & Baixo & Incerta & Alta & 01 \\
\hline Tsanakas et al. $(1983)^{24}$ & Baixo & Incerto & Baixo & Baixa & Incerta & 03 \\
\hline
\end{tabular}

QUADAS: Quality Assessment of Diagnostic Accuracy Studies. Baixo(a)=01 ponto. Alto(a)=00 pontos. Incerto(a)=00 pontos.

pediátricas, incluindo a asma, fibrose cística e as doenças neuromusculares $5,38,39$. Os achados deste trabalho mostraram que houve predomínio pela execução de três medidas de PFE ${ }^{14,16-23}$ e pela escolha do maior valor alcançado nestes registros para a interpretação dos resultados ${ }^{13,14,16-22,24}$. Isso corrobora com a recomendação da $\mathrm{ATS}^{40}$ e também está de acordo com a metodologia de outros estudos ${ }^{41-43}$, nos quais preconizam a execução de pelo menos três medidas e o uso do maior valor registrado.
Por outro lado, diverge de duas pesquisas incluídas nesta revisão, levando em conta que utilizaram a média dos registros obtidos ${ }^{15,23}$, o que pode subestimar os resultados do PFE e impactar nas equações de referência.

Pouco mais da metade dos estudos selecionados utilizou o exame clínico para a definição do status de saudável nas suas amostras 12-15,20,21,24. Estes contemplaram o exame minucioso do sistema cardiorrespiratório, tais como a ausculta pulmonar e cardíaca, inspeção da pele e 
deformidades no tórax, além de entrevista familiar. A outra parcela investigada acabou optando por avaliar o histórico de sintomas respiratórios através de questionários ${ }^{16-19,22}$, coletando a história familiar de doenças prévias, doenças cardiorrespiratórias e alérgicas, além do uso de nicotina. Segundo Esteves et al. ${ }^{44}$, o ideal é verificar se a criança/ adolescente é saudável baseado em um questionário validado, como por exemplo, o Adaptation and validity of the ATS-DLD-78. No presente estudo, somente uma pesquisa ${ }^{22}$ utilizou um questionário validado, sendo esse o ATS-DLD ${ }^{45}$. Este instrumento questiona sobre o histórico de prematuridade, doenças prévias, uso de medicações, necessidade de hospitalizações, entre outros. Isso corrobora com outros trabalhos ${ }^{45-47}$ que se propuseram a gerar equações de referência para os demais testes de função pulmonar. Portanto, nenhum estudo incluído recrutou sujeitos com doenças pré-existentes ${ }^{12-24}$.

Os estudos apresentaram qualidade metodológica adequada ${ }^{12-22,24}$, a partir da análise da escala QUADAS- ${ }^{26}$. Esta ferramenta vem sendo utilizada para averiguar o risco de viés e a preocupação quanto a aplicabilidade de outros valores de referência no âmbito respiratório e muscular ${ }^{27-29}$. Os principais fatores que baixaram a pontuação foram relacionados ao risco de viés e aplicabilidade do teste avaliado 12,13,15,16,21,23,24. Isto ocorreu devido a ausência de descrição da execução do PFE e a divergência em relação a recomendação da $\mathrm{ATS}^{40}$. Alguns estudos tiveram redução dessa análise pelo risco de viés no domínio fluxo/tempo ${ }^{14,19}$, pois excluíram/perderam participantes no momento de gerar as equações preditivas, por exemplo, na presença de outliers, perdas e divergências amostrais. Apenas o estudo de Pande ${ }^{23}$, realizado na Índia, obteve qualidade metodológica inadequada, fazendo-nos recomendar o uso das demais equações disponíveis neste país sobre o tema ${ }^{13-16,20,21}$. Embora todos os estudos apresentem amostras representativas ${ }^{12-24}$, nenhum deles testou a validade cruzada das equações, o que pode fragilizar a validação externa dos resultados. Além do mais, somente cinco deles ${ }^{12,13,16,18,20}$ relataram o poder de predição (22-76\%) e apenas dois ${ }^{21,22}$ divulgaram os erros estimados nas equações (33,2-45,1 litros/minuto), sugerindo baixo/ regular poder de explicação do PFE e considerável erro nas estimativas deste desfecho.

A exclusão de pesquisas ${ }^{48}$ que tenham gerado valores de normalidade para o PFE através de outros métodos estatísticos, torna-se a principal limitação do estudo. Nestes, podem-se listar o uso do método Linear Management System (LMS), que se baseia na interpretação dos resultados através do percentil específico para cada idade ${ }^{49}$. No entanto, a ênfase desta pesquisa foi selecionar apenas estudos de referência a partir de equações preditivas, isto é, baseadas em modelos de regressão linear. A exclusão de valores de referência para o PFE baseado na espirometria parece ser outra restrição do estudo. Porém, o uso de diferentes equipamentos pode influenciar nos resultados alcançados, o que de certo modo, inviabiliza a inclusão destes trabalhos ${ }^{50}$. Além do mais, a restrição de informações quanto às características sociodemográficas e econômicas dos trabalhos, dificultam a capacidade de extrapolação dos dados.

\section{CONCLUSÃO}

Os achados desta revisão evidenciaram que apesar da variabilidade na composição das equações de referência para o PFE, parece claro que a altura e o sexo são variáveis relevantes nos modelos de predição em diversas localidades do mundo. Até o presente momento, as fórmulas disponíveis conseguem explicar entre 18\% e $76 \%$ os resultados do PFE, obtendo as melhores predições nos trabalhos do Continente Asiático.

Interessantemente, não foram localizadas pesquisas nos continentes Oceânicos e nas Américas do Norte e Central. Além disso, o baixo poder de predição e a ausência deste item na maioria dos trabalhos incluídos ressaltam a necessidade de novas investigações no continente Europeu, na América do Sul e na África.

Espera-se que novos estudos sejam conduzidos, principalmente, nessa lacuna e que sejam levados em conta os aspectos relacionados à validade cruzada, poder de predição, além da informação quanto ao erro conhecido em cada equação.

\section{FONTE DE FINANCIAMENTO}

Nada a declarar.

\section{CONFLITO DE INTERESSES}

Nada a declarar.

\section{REFERÊNCIAS}

1. Parente AAAI, Maia PN. Testes de função pulmonar em crianças. Pulmão RJ. 2018;27(1):75-80.

2. Paes CD, Pessoa BV, Jamami M, Di Lorenzo VA, Marrara KT. Comparison between PEFvalues obtained from a population sample in the city of São Carlos, Brazil, and reference values. J Bras Pneumol. 2009 Fev;35(2):151-6. http://dx.doi. org/10.1590/S1806-37132009000200008. PMid:19287918.

3. Freitas FS, Parreira VF, Ibiapina CC. Aplicação clínica do pico de fluxo da tosse: uma revisão de literatura. Fisioter Mov. 2010;23(3):495-502. http://dx.doi.org/10.1590/S010351502010000300016.

4. II Diretrizes Brasileiras no Manejo da Tosse Crônica. J Bras Pneumol. 2006;32(Supl 6):S403-S46.

5. Fonseca AC, Fonseca MT, Rodrigues ME, Lasmar LM, Camargos PA. Peak expiratory flow monitoring of asthmatic children. J Pediatr (Rio J). 2006;82(6):465-9. http://dx.doi. org/10.2223/JPED.1566. PMid:17171206.

6. Cruz AA. Peak expiratory flow. It's better to measure! J Bras Pneumol. 2006 Jan-Fev;32(1):iv-vi. 2006;32(1):4-6. http://dx.doi.org/10.1590/S1806-37132006000100003. PMid:17278303. 
7. IV Diretrizes Brasileiras para o Manejo da Asma. J Bras Pneumol. 2006;32(Supl 7):S447-74. http://dx.doi. org/10.1590/S1806-37132006001100002.

8. Dinakar C, Oppenheimer J, Portnoy J, Bacharier LB, Li J, Kercsmar CM, et al. Management of acute loss of asthma control in the yellow zone: a practice parameter. Ann Allergy Asthma Immunol. 2014;113(2):143-59. http://dx.doi. org/10.1016/j.anai.2014.05.017. PMid:25065350.

9. BachJR, Saporito LR. Criteria for extubation and tracheostomy tube removal for patients with ventilatory failure. Chest. 1996;110(6):1566-71. http://dx.doi.org/10.1378/ chest.110.6.1566. PMid:8989078.

10. Smina M, Salam A, Khamiees M, Gada P, AmoatengAdjepong Y, Manthous CA. Cough peak flows and extubation outcomes. Chest. 2003;124(1):262-8. http://dx.doi. org/10.1378/chest.124.1.262. PMid:12853532.

11. Rodrigues JC, Cardieri JMA, Bussamra MHCF, Nakaie CMA, Almeida MB. Silva LVF Fo, et al. Provas de função pulmonar em crianças e adolescentes. J Bras Pneumol. 2002;28(Supl 3):S207-SA21.

12. Lu Y, Zheng J, Liu C, Ai T, Wang N, Meng N, et al. Peak expiratory flow among healthy children aged 5-14 years in China. J Thorac Dis. 2018;10(3):1377-85. http://dx.doi. org/10.21037/jtd.2018.02.41. PMid:29707287.

13. Kumaran K, Agrawal A. Normal Peak Expiratory Flow Rate of School Children in Jabalpur, Madhya Pradesh. Indian J Pediatr. 2017;84(8):580-4. http://dx.doi.org/10.1007/s12098017-2344-y. PMid:28389922.

14. Pramanik P, Koley D, Ganguli I. Peak expiratory flow rate in respect to anthropometric parameters of adolescent boys and girls from West Bengal, India. IOSR. 2013;13(4):58-62. http://dx.doi.org/10.9790/0853-13465862.

15. Manjunath $\mathrm{CB}$, Kotinatot SC. Peak expiratory flow rate in healthy rural school going children (5-16 years) of bellur region for construction of nomogram. J Clin Diagn Res. 2013;7(12):2844-6. PMid:24551654.

16. Taksande A, Jain M, Vilhekar K, Chaturvedi P. Peak expiratory flow rate of rural school children from Wardha district, Maharashtra in India. World J Pediatr. 2008;4(3):211-4. http:// dx.doi.org/10.1007/s12519-008-0039-1. PMid:18822931.

17. Boaventura CM, Amuy FF, Franco JH, Sgarbi ME, Matos LB. Valores de referência de medidas de pico de fluxo expiratório máximo em escolares. Arq Med ABC. 2007;32(Supl 2):S30-4.

18. Agaba PA, Thacher TD, Angyo IA, Agaba El. Peak expiratory flow rates in healthy Nigerian children. J Trop Pediatr. 2003;49(3):157-9. http://dx.doi.org/10.1093/tropej/49.3.157. PMid:12848205.

19. Høst A, Høst AH, Ibsen T. Peak expiratory flow rate in healthy children aged 6-17 years. Acta Paediatr. 1994;83(12):12557. http://dx.doi.org/10.1111/j.1651-2227.1994.tb13008.x. PMid:7734864.

20. Swaminathan S, Venkatesan P, Mukunthan R. Peak expiratory flow rate in south Indian children. Indian Pediatr. 1993;30(2):207-11. PMid:8375883.

21. Kashyap S, Puri DS, Bansal SK. Peak expiratory flow rates of healthy tribal children living at high altitudes in the Himalayas. Indian Pediatr. 1992;29(3):283-6. PMid:1612667.

22. Sanz J, Martorell A, Saiz R, Alvarez V, Carrasco JI. Peak expiratory flow measured with the Mini Wright Peak Flow Meter in children. Pediatr Pulmonol. 1990;9(2):86-90. http:// dx.doi.org/10.1002/ppul.1950090205. PMid:2144618.

23. Pande $\mathrm{AH}$. Prediction of peak expiratory flow rate from height and weight. Indian J Pediatr. 1986;53(4):521-3. http:// dx.doi.org/10.1007/BF02749540. PMid:3804393.
24. Tsanakas JN, Primhak RA, Milner RD, Hatzimichael A, Karpouzas JG. Unexpectedly high peak expiratory flow rates in normal Greek children. Eur J Pediatr. 1983;141(1):46-9. http://dx.doi.org/10.1007/BF00445668. PMid:6641765.

25. Moher D, Liberati A, Tetzlaff J, Altman DG, The PRISMA Group. Preferred reporting items for systematic reviews and meta-analyses: the PRISMA statement. PLoS Med. 2009;6(7):e1000097. http://dx.doi.org/10.1371/journal. pmed.1000097. PMid:19621072.

26. Whiting PF, Rutjes AW, Westwood ME, Mallett S, Deeks JJ, Reitsma JB, et al. QUADAS-2: a revised tool for the quality assessment of diagnostic accuracy studies. Ann Intern Med. 2011;155(8):529-36. http://dx.doi.org/10.7326/0003-4819155-8-201110180-00009. PMid:22007046.

27. Sclauser Pessoa IM, Franco Parreira V, Fregonezi GA, Sheel AW, Chung F, Reid WD. Reference values for maximal inspiratory pressure: a systematic review. Can Respir J. 2014;21(1):43-50. PMID: 24137574.

28. Benfica PDA, Aguiar LT, Brito SAF, Bernardino LHN, TeixeiraSalmela LF, Faria CDCM. Reference values for muscle strength: a systematic review with a descriptive metaanalysis. Braz J Phys Ther. 2018;22(5):355-69. http://dx.doi. org/10.1016/j.bjpt.2018.02.006. PMid:29764761.

29. Verma R, ChiangJ, Qian H, Amin R. Maximal static respiratory and sniff pressures in healthy children. a systematic review and meta-analysis. Ann Am Thorac Soc. 2019;16(4):47887. http://dx.doi.org/10.1513/AnnalsATS.201808-506OC. PMid:30562038.

30. Ozarda Y. Reference intervals: current status, recent developments and future considerations. Biochem Med (Zagreb). 2016;26(1):5-16. http://dx.doi.org/10.11613/ BM.2016.001. PMid:26981015.

31. Pasquali L. Validade dos testes psicológicos: será possível reencontrar o caminho? Psic Teor Pesq (online). 2007;23(esp):099-107.

32. Miller A, American Thoracic Society. Lung function testing: selection of reference values and interpretative strategies. American Thoracic Society. Am Rev Respir Dis. 1991;144(5):1202-18. http://dx.doi.org/10.1164/ ajrccm/144.5.1202. PMid:1952453.

33. Schivinski CIS, Gonçalves RM, Castilho T. Valores de referência para força muscular respiratória em crianças brasileiras. J Hum Growth Dev. 2016 Nov 28;26(3):374-9. http://dx.doi.org/10.7322/jhgd.122912.

34. Fritscher CC. Pico de fluxo expiratório em escolares de Porto Alegre (RS): proposta para valores de referência. Rev Med PUCRS. 1996;6(4):19-23.

35. Wall MA, Olson D, Bonn BA, Creelman T, Buist AS. Lung function in North American Indian children: reference standards for spirometry, maximal expiratory flow volume curves, and peak expiratory flow. Am Rev Respir Dis. 1982;125(2):158-62. PMid:7065517.

36. Takase M, Sakata H, Shikada M, Tatara K, Fukushima T, Miyakawa T. Development of reference equations for spirometry in Japanese children aged 6-18 years. Pediatr Pulmonol. 2013;48(1):35-44. http://dx.doi.org/10.1002/ ppul.22536. PMid:22431482.

37. Marcondes E. Pediatria básica. São Paulo: Sarvier; 1991.

38. Ramos FAB, Ordonho MC, Pinto TCVR, Lima CA, Vasconcelos CR. Evaluation of the respiratory muscle force and the peak flow in patients with Duchenne muscular dystrophy exposed to non-invasive ventilation and hydrotherapy. Pulmão RJ. 2008;17(2-4):81-6.

39. Gauld LM, Briggs K, Robinson P. Peak inspiratory flows in children with cystic fibrosis. J Paediatr Child Health. 
2003;39(3):210-3. http://dx.doi.org/10.1046/j.14401754.2003.00127.x. PMid:12654145.

40. Miller MR, Hankinson J, Brusasco V, Burgos F, Casaburi R, Coates A, et al. Standardisation of spirometry. Eur Respir J. 2005;26(2):319-38. http://dx.doi.org/10.1183/09031936.05 .00034805 . PMid:16055882.

41. França DC, Camargos PA, Jones MH, Martins JA, Vieira BS, Colosimo EA, et al. Prediction equations for spirometry in four- to six-year-old children. J Pediatr (Rio J). 2016 Jul-Ago;92(4):400-8. http://dx.doi.org/10.1016/j. jped.2015.10.005. PMid:27161560.

42. Vilozni D, Barak A, Efrati O, Augarten A, Springer C, Yahav $Y$, et al. The role of computer games in measuring spirometry in healthy and asthmatic preschool children. Chest. 2005;128(3):1146-55. http://dx.doi.org/10.1378/ chest.128.3.1146. PMid:16162700.

43. Cole TJ, Stanojevic S, Ações J, Coates AL, Hankinson JL, Wde AM. Relacionado a idade e tamanho intervalos de referência: um estudo de caso da espirometria na infância e na idade adulta. Estatística em Medicina. 2009;28(5):880-98.

44. Esteves A, Solé D, Ferraz MB. Adaptation and validity of the ATS-DLD-78-C questionnaire for asthma diagnosis in children under 13 years of age. Braz Ped News. 1999;1:3-5.

45. Heinzmann-Filho JP, Vidal PCV, Jones $M H$, Donadio MVF. Normal values for respiratory muscle strength in healthy preschoolers and school children. Respir
Med. 2012;106(12):1639-46. http://dx.doi.org/10.1016/j. rmed.2012.08.015. PMid:22974829.

46. Woszezenki CT, Heinzmann-Filho JP, Vendrusculo FM, Piva TC, Levices I, Donadio MV. Reference values for inspiratory muscle endurance in healthy children and adolescents. PLoS One. 2017;12(1):e0170696. http://dx.doi.org/10.1371/ journal.pone.0170696. PMid:28122012.

47. Jones MH, Vidal PCV, Lanza FC, Silva DCFMF, Pitrez PM, Olmedo APBF, et al. Reference values for spirometry in Brazilian children. J Bras Pneumol. 2020 Mar 27;46(3):e20190138. http://dx.doi.org/10.36416/1806-3756/ e20190138. PMid:32236343.

48. Cossio-Bolaños MA, Andruske C, Arruda M, Sulla-Torres J, Pacheco-Carrillo J, Urra-Albornoz C, et al. Reference norms for evaluating maximum expiratory flow of children and adolescents of the Maule Region in Chile. PeerJ. 2018 Jul 16;6:e5157. http://dx.doi.org/10.7717/peerj.5157. PMid:30038857.

49. Cole TJ. The LMS method for constructing normalized growth standards. Eur J Clin Nutr. 1990;44(1):45-60. PMid:2354692.

50. Pesola GR, O'Donnell P, Pesola GR Jr, Chinchilli VM, Saari AF. Peak expiratory flow in normals: comparison of the mini Wright versus spirometric predicted peak flows. J Asthma. 2009;46(8):845-8. http://dx.doi. org/10.1080/02770900903159726. PMid:19863291. 